\title{
Sensitized Patients, Transplant, and Management
}

\author{
Hariharan S. Iyer • Annette M. Jackson • \\ Robert A. Montgomery
}

Published online: 7 March 2014

(C) Springer International Publishing AG 2014

\begin{abstract}
Sensitization to human leukocyte antigens (HLA) creates significant barriers for renal transplant candidates, reducing access to transplantation by limiting donor availability and increasing the risk of rejection. Sensitized patients also endure longer wait-times on dialysis. Desensitization protocols, kidney-paired donation, and a combination of both of these modalities have been used to remove or circumvent the clinically deleterious anti-HLA antibodies. Technical advances in antibody characterization methods and therapies targeting the humoral arm of the immune system have facilitated successful transplantation for these immunologically challenging patients. However, antibody-mediated rejection and transplant glomerulopathy resulting from injury to the vascular endothelium are major impediments to further improvement in outcomes. This review describes the assessment protocols, antibody monitoring techniques, and therapeutic strategies that have been used effectively to overcome HLA incompatibility.
\end{abstract}

\footnotetext{
H. S. Iyer

Department of Medicine, Division of Nephrology and

Transplantation, London Health Sciences Center,Schulich School of

Medicine and Dentistry, Western University, Room ALL-139,

University Hospital, 339 Windermere Road, London, Ontario,

Canada N6A5A5

e-mail: hariharan.iyer@lhsc.on.ca

A. M. Jackson

Department of Medicine, Associate Director, Immunogenetics

Laboratory, Johns Hopkins University School of Medicine, 2041 E.

Monument Street, Baltimore, MD 21205-2222, USA

e-mail: ajackson@jhmi.edu

R. A. Montgomery $(\bowtie)$

Division of Transplant Surgery, Johns Hopkins University School of Medicine, 720 Rutland Avenue, Ross 765, Baltimore, MD 21205, USA

e-mail: rmonty@jhmi.edu
}

Keywords Kidney-paired donation · Desensitization · Complement inhibition · Donor-specific antibodies · Cytotoxic crossmatch $\cdot$ Panel-reactive antibody $\cdot$ Solid-phase immunoassay $\cdot$ Plasmapheresis $\cdot$ Intravenous immunoglobulin $\cdot$ Transplant $\cdot$ Management

\section{Introduction}

Kidney transplantation is the treatment of choice for patients with end-stage renal disease. Despite efforts to increase livingdonor transplantation, many potential donor-recipient pairs are deemed incompatible due to the presence of preformed antibodies against donor human leukocyte antigens (HLA). Nearly one-third of patients on the wait-list are sensitized to HLA, and around $15 \%$ have calculated panel-reactive antibodies $($ CPRA $)>80 \%$ [1]. These difficult-to-match recipients are disadvantaged by extremely low transplant rates and are relegated to the ever-expanding deceased donor pool. By markedly prolonging wait-times and increasing the cost of care, sensitization to HLA imposes a huge economic burden on the health care system and constitutes a major public health problem.

Innovative strategies such as desensitization, kidney-paired donation and the use of new therapeutic agents have enabled transplant centers to overcome this immunological barrier. These strategies have been enhanced by sensitive and specific assays that permit identification of antibody specificities with high resolution, permitting better characterization of donorrecipient phenotypes.

\section{Sensitization to HLA After Alloimmunization}

Sensitization to allogeneic HLA occurs as a consequence of prior transplantation, pregnancy, or the receipt of blood products. Despite the high degree of polymorphism within the 
HLA system, exposure to public epitopes shared between common and less common antigens can result in a broadened level of sensitization. Development of de novo anti-HLA donor-specific antibodies (DSA) has an adverse impact on allograft outcomes [2]. Our understanding of the alloimmune response toward HLA is far from complete, and it is not entirely clear as to why some individuals develop antibodies following a single allosensitizing event while others do not. In addition to the creation of antibodies specific to the exposed HLA, some patients experience increased breadth of antibody reactivity against "public" epitopes shared between polymorphic HLA molecules. Porcheray et al. provided further insight into this phenomenon by analyzing B-cell clones from a human renal allograft undergoing humoral rejection and characterizing the antibody specificities. They demonstrated the formation of polyreactive antibodies with apparent reactivity against HLA and multiple auto-antigens [3]. These polyreactive antibodies may be the key to understanding the astounding rise in CPRA following sensitization in some individuals that cannot be construed based purely on frequency of the genotype or known public epitopes.

\section{Role of the Histocompatibility Laboratory}

The histocompatibility laboratory is a critical component of the multidisciplinary team involved in the care of immunologically complex transplant patients.

\section{Defining Unacceptable Antigens and Predicting the Virtual Crossmatch}

While the concept of a virtual crossmatch has been in existence for decades, advancements in methods of antibody detection and specificity characterization have greatly improved the accuracy of these predictions. Initially, the virtual crossmatch was limited to HLA antibodies strong enough to yield a positive cytotoxicity crossmatch, and these HLA mismatches were deemed unacceptable. Unacceptable antigens were defined using cytotoxicity screening to test the recipient's sera against lymphocytes from a panel of donors selected to represent a wide array of HLA. The number of positive reactions was used to derive a panel-reactive antibody percentage (\%PRA) that provided clinicians with information regarding the breadth of HLA sensitization and the likelihood of finding a crossmatch negative donor. Cytotoxicity methods have been limited due to their insensitivity and interference from irrelevant autoantibodies. Today, the task of defining unacceptable antigens is complicated by the inherent differences among transplant programs with respect to the level of DSA that is considered unacceptable [4]. While newer CPRA values continue to provide information regarding the breadth of sensitization, they no longer provide details on the antibody strength.
Solid-phase immunoassay (SPI) platform options - ELISA or beads analyzed with flow cytometry or Luminex technology - vary in both sensitivity and equipment requirements. In addition, binding recombinant HLA to solid matrices has been shown to distort the architecture of native epitopes or at times to express cryptic epitopes, resulting in false-negative and false-positive reactions, respectively [5,6]. Given these shortcomings, there is a compelling need for each transplant program to correlate the results of SPI with crossmatch results and clinical outcomes. We have previously shown that the strength of DSA reactivity determined by phenotype-panel SPI correlates significantly with the strength of T- and B-cell cytotoxic $(\mathrm{r}=0.83)$ and flow-cytometric crossmatch $(\mathrm{FCXM})$ $(r=0.85)$ tests. These correlations have enabled us to generate threshold values that correctly predict cytotoxic and FCXM results with high frequency ( $93 \%$ of cases), improving the validity and reliability of the virtual crossmatch [7].

\section{Quantifying Antibodies}

There are differences among histocompatibility laboratories with regard to the procedures used for crossmatch testing and thresholds that define positivity. In a FCXM, the difference between the median channel fluorescence of the test serum and that of the negative control serum, expressed as a channel shift or ratio, is used to determine the presence and quantity of alloantibody bound to donor lymphocytes. While it is a popular tool, the FCXM is influenced by numerous physiological and technical factors, including the amount of antigen expression on the cells, the detection antibody used, cell and serum treatments to reduce background reactivity, and instrument calibration [8]. Using a ratio rather than channel-shift may rectify some of these anomalies and achieve better correlations with clinical outcomes [9]. Molecular equivalents of soluble fluorochrome (MESF) or standardized fluorescence intensity (SFI) can also be determined from standard curves to improve the reproducibility of the test results [8].

\section{Reporting Sensitization}

Several techniques have been used to identify antigens against which the patient has an unacceptable level of reactivity. Eurotransplant uses an approach that involves running multiple cross-match tests against donors mismatched only for a single HLA. This requires access to viable cells from a large pool of HLA-typed individuals. In the U.S., the CPRA policy governs reporting sensitization and kidney-pancreas organ allocation within the United Network for Organ Sharing (UNOS) [10, 11]. Information on anti-HLA antibodies characterized by SPI is entered into an online program to generate the frequency of donors with unacceptable antigens. Sensitized patients with CPRA $\geq 80$ are awarded extra allocation points to facilitate transplantation [12-14]. 
The CPRA calculator is available at: http://optn.transplant. hrsa.gov/resources/professionalResources.asp?index $=78$ ).

\section{Eliminating Interference from Solid-Phase Immunoassays}

When using SPI platforms, naturally occurring factors such as IgM antibodies, immune complexes, and non-specific binding to beads can increase the background reactivity and interfere with the test results. Serum treatments such as dithiothreitol (DTT) and hypotonic dialysis (HD) have been used as a corrective measure. In HD, serum is dialyzed against distilled water, resulting in precipitation and effective elimination of the water-insoluble IgM molecules [15]. Zachary et al. demonstrated that while HD increased the reaction strength of positive control beads and reduced the reaction strength of negative control beads, DTT increased the reactivity of both. HD also improved the determination of DSA specificity and crossmatch predictability to a greater extent than DTT, leading to more meaningful test interpretation [16].

\section{Establishing the most Likely Route to Successful Transplantation}

Sensitized patients have a lower likelihood of being offered a crossmatch negative donor. As a result, these patients languish on waiting lists. Various strategies have been employed to resolve this issue. First, desensitization protocols are applied to lower the antibody strength to an acceptable level pretransplantation. Second, an attempt is made to avoid the unacceptable HLA against which the patient has antibodies through kidney-paired donation (KPD). For deceased-donor transplantation, patients are awarded extra allocation points and are enrolled into special programs such as the Acceptable Mismatch Program of Eurotransplant [17]. The most suitable approach is usually dictated by immunologic phenotyping to determine the specificity and strength of the patient's antiHLA antibodies.

\section{Desensitization}

Desensitization protocols are not standardized and are centerspecific. Similarly, the threshold of antibody strength and reactivity that is acceptable for desensitization must be determined individually by each program after taking into account factors such as local expertise, resource availability, and clinical outcomes.

The Johns Hopkins protocol for living-donor crossmatch positive desensitization is based on a combination of plasmapheresis (PP) and low-dose intravenous immunoglobulin (IVIg) $(100 \mathrm{mg} / \mathrm{kg})$. The initial DSA strength is used to determine the number of pre-transplant sessions. The goal prior to surgery is to reduce the DSA strength to below a positive cytotoxic crossmatch level (roughly equivalent to mean fluorescent intensity [MFI] scores of 10,000 on a phenotype-panel for most antigens in our laboratory) or to produce a sustained reduction if initial antibody strength is below a cytotoxic level (Fig. 1). Treatment is continued postoperatively until the DSA strength is below a positive FCXM level (around $6000 \mathrm{MFI}$ ).

Anti-CD20 is used preoperatively in a select group of patients (multiple repeat mismatches, very high DSA titers, or increased levels of HLA-specific B-cells on tetramer staining). Sustained elimination of DSA with this protocol is influenced by the DSA titer, class of HLA, and epitope (private vs. public) to which it is directed. Seventy-five percent of class I HLA antibodies, $53 \%$ of antibodies specific for DRB1 and DQB antigens, and only $10 \%$ of antibodies to DR51, DR52, and DR53 are eliminated. DSAs with reactivity to only one member of a cross-reactive group (CREG) (private epitopes) are much more refractory to treatment $(70.8 \%$ persist $)$ than those reactive with several members of the CREG (public epitopes) (26.5\% persist) [18].

Protocol biopsies are conducted at 1, 3, 6, and 12 months. The rate of clinically apparent antibody-mediated rejection (AMR) in our cohort is approximately $22 \%$; most of these episodes respond to retreatment with PP/IVIg [19•]. To reduce the risk of AMR, it is important to identify and characterize all antibody specificities, including those to HLA-DQA, DPA, and to epitopes that consist of portions of the $\alpha$ and $\beta$ chains of DQ and DP molecules [20].We recently reported on the time course of histologic allograft features in HLAincompatible transplant recipients. Microcirculation inflammation, particularly glomerulitis, was identified as an independent risk factor for the development of transplant glomerulopathy at 1 year [21].

\section{Outcomes of Desensitization}

Graft and patient survival rates have varied in single-center studies, but are generally considered to be inferior to compatible living-donor renal transplants [22, 23]. A negative pretransplant cytotoxic crossmatch confers better medium-term survival than one that is positive [24]. Outcomes of desensitization protocols should be weighed against options that are actually achievable for the patient. For a broadly sensitized patient with very high-titer anti-HLA antibodies, receiving a crossmatch negative compatible kidney is not a realistic option. Other than desensitization, the only alternative for these patients is waiting on the deceased donor list for the rare compatible organ. In a detailed analysis on the survival of 211 patients undergoing desensitization, our group demonstrated that, independent of the strength of DSA, HLAincompatible transplantation conferred significant survival benefit. The 8 -year patient survival was $80.6 \%$, while the rate for the matched controls waiting on dialysis for a compatible organ was $49.1 \%(\mathrm{P}<0.001)[25 \bullet \cdot$. Only $16 \%$ of the 


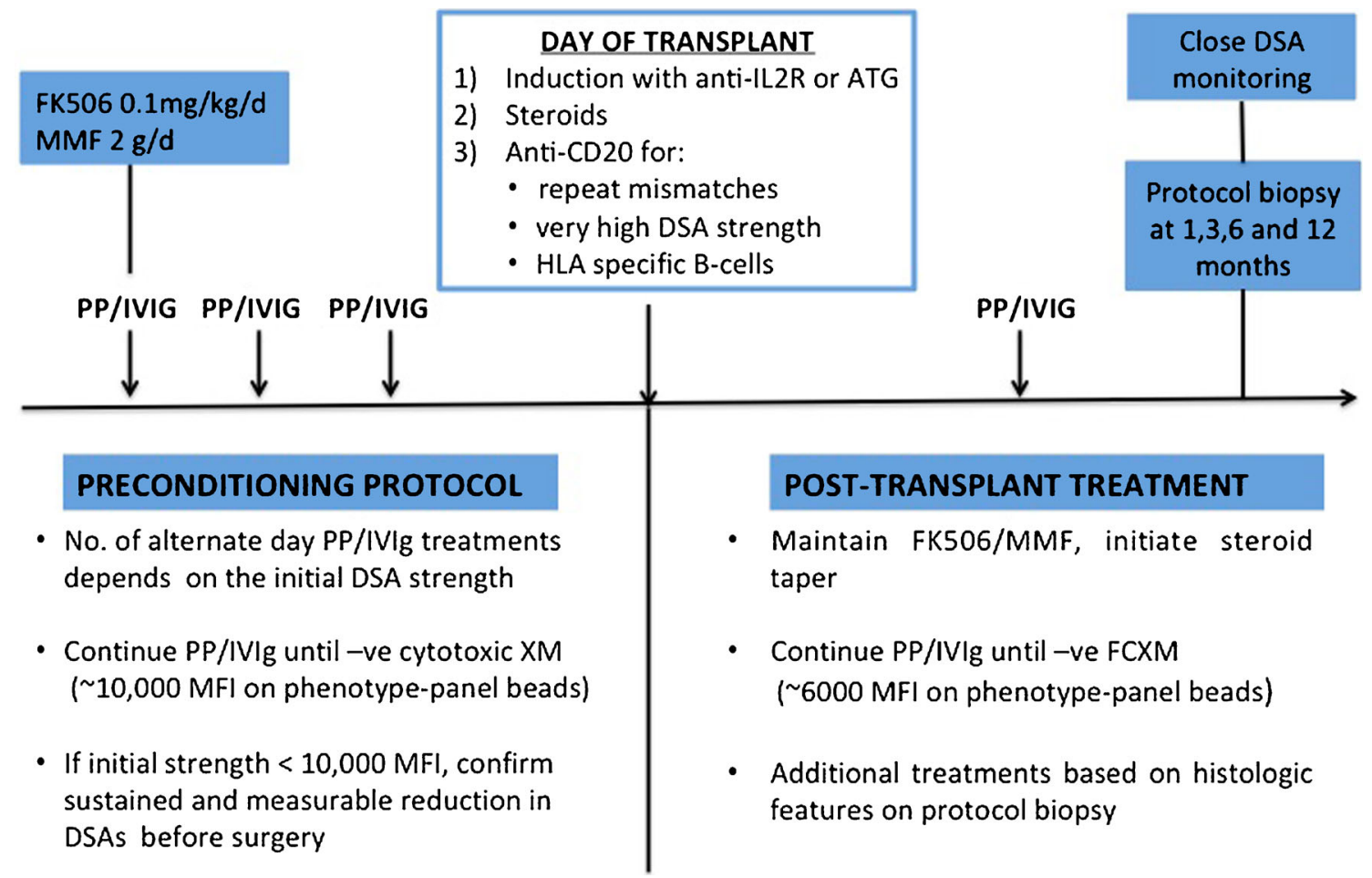

Fig. 1 The Johns Hopkins protocol for HLA-incompatible transplantation. ATG, antithymocyte globulin; DSA, donor-specific antibody; FCXM, flow-cytometric crossmatch; FK506, tacrolimus; IVIg,

matched controls were transplanted, indicating that maintenance dialysis is the only realistic option for the vast majority of sensitized patients. The survival of matched-control patients who remained on dialysis during the 8 -year follow-up period was only $30.5 \%$.

In a multicenter randomized placebo-controlled trial led by the Cedars-Sinai group, an alternative strategy comprising monthly infusions of $2 \mathrm{~g} / \mathrm{kg}$ of IVIg (up to 4 doses) demonstrated a significant reduction in PRA and higher transplantation rates ( 39 vs. $17 \%, \mathrm{n}=101$ ) [26]. Addition of anti-CD20 to this protocol achieved transplantation rates of $80 \%$, with good short-term outcomes [27]. A potential strategy for patients awaiting deceased-donor transplantation is to reduce antibody strength by preemptively treating the patient with the highdose IVIg protocol, with or without anti-CD20. The PP/IVIg protocol may be implemented postoperatively to further lower the level of DSA and subsequent risk of AMR. The combination of anti-CD20 and high-dose IVIg appears to be more efficacious in reducing antibodies of lower strength [28, 29].

\section{Kidney-Paired Donation}

Kidney-paired donation (KPD), which involves the exchange of kidneys between incompatible pairs so that each recipient receives a compatible organ, is an attractive option for highly sensitized patients. The chief limitations of KPD result from blood-group imbalance as well as competition for rare HLA intravenous immunoglobulin; MMF, mycophenolate mofetil; PP, plasmapheresis; XM, crossmatch

genotypes for highly sensitized patients. Blood-type skewing results from $\mathrm{O}$ recipients being overrepresented in KPD pools with inadequate numbers of $\mathrm{O}$ donors. While mathematical simulations have indicated an overall match rate of approximately $50 \%$ for a typical national KPD pool, match rates are considerably lower for certain donor/recipient phenotypes: $17 \%$ for A-to-O blood type incompatible pairs and $14 \%$ for highly sensitized patients who have an O donor [30॰]. Eventually, the KPD pool becomes overwhelmed with an increasing population of these difficult-to-match candidates [31]. Match rates may be considerably increased by (up to $75 \%$ ) including compatible pairs in order to add normative blood types to the KPD pool. Expanding the genetic diversity of the KPD pool is another strategy to increase match rates. With a view toward the optimization and expansion of the KPD spectrum, we recently reported on a 10-way domino transplant that involved the exchange of kidneys between the United States and Canada [32].

\section{Combined Kidney-Paired Donation and Desensitization}

As a general principle, patients with a narrow breadth of sensitization but high-titer DSAs will benefit from KPD. Desensitization protocols are best applied to broadly sensitized patients, particularly those with rare phenotypes or homozygosity at any HLA locus. Desensitization is also a good option for highly sensitized patients who have low-level DSA. 
Unfortunately, some broadly sensitized patients also have very high levels of HLA reactivity, which precludes both desensitization and KPD. In such cases, it may be possible to find a donor from the KPD pool against whom the patient has lowlevel antibodies that can be effectively eliminated by desensitization [33]. In this scenario DSA below a certain threshold (for instance, a positive FCXM strength) would be removed from the unacceptable antigen list, and the KPD pool would be searched for a suitable genotype that would include these antigens [34]. The low-level DSA that would likely be present is then removed by desensitization. This concept is illustrated in Fig. 2. Numerous patient-specific, donor-specific, and program-specific factors may be utilized to generate collective data that can be incorporated into a generic Bayes' calculation to estimate the likelihood of achieving transplantation. With this methodology, we have reported a nearly $95 \%$ probability of transplantation using one of these options [35••].

\section{DSA Monitoring After Transplantation}

It is important to distinguish antibodies that are detected pretransplant from those that develop de novo following transplantation. Unfortunately, the large body of literature in this field is conspicuously lacking a rigorous definition of preexisting versus de novo DSAs. In a retrospective study by Amico et al., nearly $50 \%$ of patients who had DSAs that were not recognized pre-transplant went on to develop AMR and had a significantly lower graft survival $(n=34, P<0.0001)$. MFI values and the antibody specificity (class I vs. class II) did not reliably predict the risk of AMR [36]. The Mayo group examined patients who had pre-transplant DSAs at varying levels (FCXM $<300$, FCXM $>300$, +ve cytotoxic-AHG XM) and were desensitized using PP/IVIg. They found that the risk

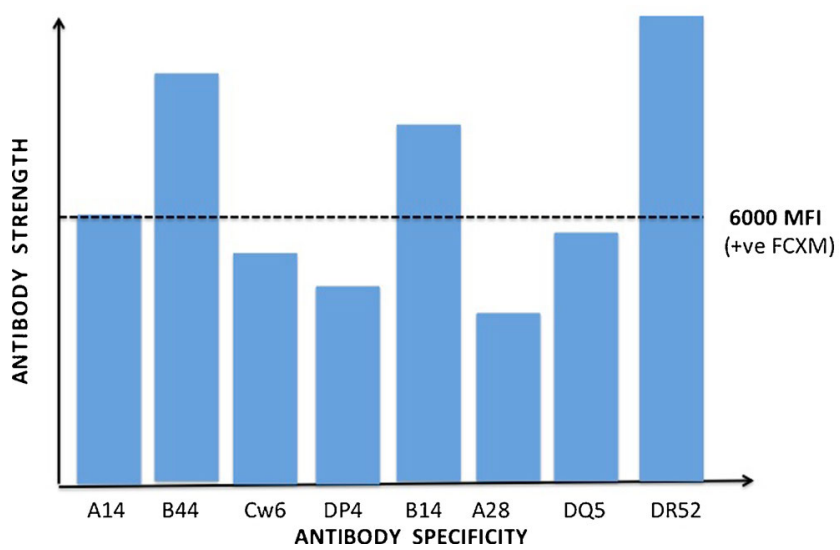

Fig. 2 If all of the antibody specificities are disallowed, none of them would be considered acceptable. If the threshold is raised to a level that produces a positive flow crossmatch $(6000 \mathrm{MFI})$, specificities below that threshold i.e. Cw6, DP4, A28, and DQ5 can be overcome using desensitization protocols. These are entered into the KPD algorithm as acceptable antigens and a search is carried out for a suitable genotype to avoid the remaining specificities (B44, B14, DR52) of AMR correlated with the strength of the FCXM, and much less so with MFI values [37]. The Edmonton group reported that in patients who had a biopsy for a clinical indication, the time of onset of DSAs relative to the onset of graft dysfunction could not be established $(n=145)$ [38]. In a single-center cohort study, Wiebe et al. sequentially evaluated the sera of low-risk renal transplant recipients and demonstrated that the frequency of de novo DSAs progressively increased with time. Fifteen percent of patients developed anti-HLA DSAs at a mean of $4.6 \pm 3.0$ years post-transplantation $(n=315)$ [39].

Mismatches at the HLA-DRB1 locus, non-adherence to medications, and clinical or subclinical episodes of cellular rejection were identified as independent risk factors for the development of de novo DSAs. Some patients with de novo DSAs follow an indolent course, with relatively stable graft function. Factors such as initial antibody titer, complementfixing ability, IgG subclass, differences in epitope immunogenicity or binding avidity, and the modification of immune responses by regulatory $\mathrm{T}$ - or B-cells may explain the lack of dysfunction in this group. A consensus meeting was convened in Rome recently that brought together experts from around the world to provide state-of-the-art guidance for antibody monitoring. The guidelines that emerged from this meeting recommend that DSAs should be closely monitored and a protocol biopsy conducted in the first three months for highly sensitized patients. Evidence of clinical or subclinical rejection or rising DSA levels should prompt initiation of therapy $\left[40^{\bullet}\right]$.

The first few weeks post-transplantation represent a critical phase in the alloimmune response and one that requires careful monitoring. Recognizing the significance of a rising test value on SPI is critical in decision-making. A considerable rise in DSA titer usually epitomizes a real increase and warrants prompt intervention. Smaller increases in a Luminex ${ }^{\circledR}$ bead assay could represent day-to-day variability in test sensitivity. In such cases, we have found that comparing the reading for the beads bearing the donor antigen with the positive control value can be very useful. Another approach is to evaluate the ratio of the test bead value to the positive control value $[41 \bullet \bullet]$.

\section{Emerging Therapeutic Options}

\section{Proteasome Inhibition}

Bortezomib, a first-in-class proteasome inhibitor, is a chemotherapeutic agent that targets plasma cells and is used for the treatment of multiple myeloma. Research was undertaken to explore its potential to reverse humoral rejection and achieve a sustained reduction in DSA levels in sensitized dialysis patients [42•], but the patients in this trial were also receiving other DSA-depleting therapies, including plasmapheresis and IVIg, and the relative contribution of bortezomib to the 
therapeutic efficacy could not be established. Studies have demonstrated its ability to induce apoptosis of bone marrowderived human plasma cells, curtail plasma cell longevity, and interfere with the antibody-producing mechanism [43]. Using pre- and post-treatment ELISPOT assays, the Mayo group demonstrated that bortezomib effectively depleted DSAproducing plasma cells (DSA-PC) $(6.7 \pm 14.5 \mathrm{DSA}-\mathrm{PCs} / \mathrm{ml}$ pre-treatment vs. $6.2 \pm 3.6 \mathrm{DSA}-\mathrm{PCs} / \mathrm{ml}$ post-treatment, $\mathrm{n}=8$, $\mathrm{P}=0.048$ ) and reinforced the efficacy of antibody removal using plasma exchange [44]. More recently, the Cincinnati group conducted a pilot study to evaluate the safety and toxicity profile of a protocol that combined anti-B-cell therapy (bortezomib and rituximab) with a T-cell-depleting agent (rabbit anti-thymocyte globulin) for induction in high-risk renal transplant recipients $(n=40)$. There were no serious toxicities or deaths from infection or malignancy [45]. Further investigation is warranted to determine if the addition of B-celldepleting agents would enhance the effects of T-cell-based therapy and improve outcomes in sensitized patients.

\section{Therapies Directed Towards the Complement Pathway}

Activation of the complement cascade plays an integral role in the pathogenesis of alloantibody-mediated graft injury. [46]. Eculizumab (Alexion, Cheshire, Connecticut), a humanized monoclonal antibody against complement factor $\mathrm{C} 5$, blocks the cleavage of C5 into C5a and C5b, inhibits the formation of membrane attack complex, and prevents the amplification of the complement system. Eculizumab has been used in renal transplant recipients to treat atypical HUS and as prophylaxis to prevent its recurrence. Its utility has been expanded of late to treat other disorders of complement dysregulation such as C3-glomerulopathy, dense-deposit disease, and thrombotic microangiopathy related to catastrophic antiphospholipidantibody syndrome [47, 48]. The first reported use of eculizumab in the kidney transplant population was from our group, in which the drug successfully reversed severe AMR [49]. Stegall et al. reported on their experience using eculizumab post-transplantation in high-risk patients desensitized with PP/IVIg. Treatment with eculizumab resulted in a marked reduction in AMR $(7.7 \%$ vs. $41 \%, p=0.003$, $\mathrm{n}=26$ ) and a lower rate of transplant glomerulopathy at one year $(6.7 \%$ vs. $35.7 \%, p=0.04)[50 \bullet \cdot]$.

Several clinical phenotypes of AMR have been recognized based on the mechanism of antibody production and the timing of injury post-transplantation. Accelerated AMR triggered by an anamnestic memory B-cell response usually occurs in the first week and is characterized by acute graft dysfunction, thrombocytopenia, oliguria, rapidly rising DSA titers, and histologic features of thrombotic microangiopathy and coagulative necrosis. This entity does not respond well to standard treatment protocols. In the past, splenectomy has been used with a greater than $85 \%$ success rate $[51,52]$.
Combining eculizumab and splenectomy may provide further improvement in outcomes for early severe AMR after desensitization. The optimum duration of therapy is yet to be ascertained. The role of proximal complement inhibition using $\mathrm{C} 1$ esterase inhibitor in reversing this AMR phenotype in patients undergoing HLA-incompatible transplantation is currently being investigated (NCT01134510).

\section{Novel Diagnostic Approaches}

\section{Functional C1q Assay}

HLA molecules on the vascular endothelium of the allograft constitute the first point of contact for DSA. Complementfixing DSA binds C1q to activate the classical complement pathway, producing a distinct pattern of injury that is characteristic of AMR. Chen and colleagues developed the C1q assay to identify and define DSA phenotypes with complement-fixing capability on solid-phase beads [53]. They also showed that $\mathrm{Clq}$ binding confers pathogenicity to DSA, which translates into a greater risk of allograft loss [54]. Loupy et al. recently substantiated these findings through a large population-based cohort study [55*0]. At one year, C1qbinding DSAs were associated with an increased frequency of AMR, more severe microcirculation inflammation on histology, more intense $\mathrm{C} 4 \mathrm{~d}$ deposition in the peritubular capillaries, and a lower eGFR $(\mathrm{n}=1016)$. C1q binding also correlated with poor graft survival at five years. Patients with C1qbinding DSA had higher strength of antibody reactivity (MFI $\geq 6000$ ) than those with non C1q-binding DSA, which may be a confounding factor in this study.

Although the data presented here are enticing, several aspects require further clarification. Non-HLA antibodies that are known to cause microvascular injury through complement-independent mechanisms, as well as low-titer anti-HLA DSAs that individually do not fix-complement, remain undetected by this assay. Given the relatively short follow-up period, slowly progressive microvascular injury resulting from DSAs against class-II HLA may have been underappreciated by this study [56]. Prospective trials are needed to examine the response of C1q-binding DSA to antibody-depleting therapies.

\section{Identification of Non-HLA Antibodies}

In recent years, features resembling AMR have been reported in patients receiving a blood type-compatible kidney from an HLA-identical sibling, indicating the involvement of antigenic targets other than HLA and ABO blood-type in the alloimmune response [57]. These antibodies may escape detection with conventional crossmatch techniques that utilize lymphocytes as target cells. A novel flow-cytometric assay $\left(\mathrm{XM}-\mathrm{ONE}{ }^{\circledR}\right.$, Absorber) that uses endothelial precursor cells 
(EPC) as targets has been developed to detect alloreactive anti-endothelial cell antibodies (AECA) [58]. With this technique, EPCs are isolated from the peripheral blood of the donor using anti-Tie2 magnetic nanoparticles and positive selection. Tie 2 is a tyrosine kinase expressed on the surface of EPCs [59]. It is important to recognize the varying clinical phenotypes of injury associated with AECA. We have shown that antibodies directed against endothelial cell antigens are enriched for non-complement-fixing subclasses IgG2 and IgG4, rendering strategies to combat complement-fixing antibodies ineffective (e.g., complement inhibitors) [60••]. While several studies have reported on the correlation between IgGAECAs detected by the XM-ONE ${ }^{\circledR}$ assay and an increased frequency of rejection and graft dysfunction [61, 62], others have raised questions about its utility and predictive value [63, 64].

Angiotensin II type I receptor (AT1R) has been identified as one of the antigenic targets for AECAs. Activating antibodies to AT1R have been implicated in the pathogenesis of AMR and obliterative microvasculopathy after renal and cardiac transplantation $[65,66]$. In a recent publication, Giral et al., using a newly developed solid-phase assay, identified humoral pre-sensitization to AT1R as an independent risk factor for the development of acute renal allograft rejection $(\mathrm{HR}=1.91,95 \% \mathrm{CI}=[1.03,3.54], \mathrm{p}=0.0393, \mathrm{n}=599)$ and long-term graft loss [67]. We have previously reported on the association between antibodies to AT1R and a phenotype characterized by AMR and de novo glomerular disease [68•].

\section{Quantifying Cryptic Sensitization to HLA}

Many patients sensitized to HLA may not have detectable antibodies at the time of testing. Once formed, antibodies persist for years in some patients, whereas in others they are short-lived. HLA tetramers are complexes comprised of four peptide-loaded HLA molecules, with a fluorescently labeled streptavidin molecule forming the centerpiece. Traditionally used to examine T-cell-dependent cell-mediated immune response, tetramer staining has recently been deployed to detect, isolate, and enumerate HLA-specific B-cells in sensitized patients [69, 70], and has been utilized to predict posttransplant anamnestic response in patients for whom details regarding allosensitizing events are undocumented or not clearly defined. Patients that are positive for tetramer staining are more likely to rapidly develop alloreactive antibodies to the specific HLA molecule, either on repeated exposure to the antigen or from non-specific activation of polyclonal B-cells from a proinflammatory response to surgery [71]. We have demonstrated that eliminating HLA-specific memory B-cells with anti-CD20 therapy can prevent such an anamnestic response. In the tetramer-positive group $(n=26)$, none of the patients treated with rituximab formed DSA to the tetramer antigen. In contrast, $81.2 \%$ of untreated patients mounted an alloimmune response post-transplantation $(\mathrm{P}=0.00006)$ [72].

Future Directions and Research

While considerable progress has been made in developing treatment strategies and improving outcomes for patients sensitized to HLA, major challenges still remain. Concrete efforts must be directed toward achieving standardization of test results and comparability between histocompatibility laboratories. Clinical trials exploring sequential monitoring to correlate antibody levels with histologic response to therapy and long-term outcomes are warranted. Further investigation is needed to evaluate the kinetics of antibodies with complement-fixing ability, their response to therapy, and the ramification of therapy on clinical endpoints. Finally, the holy grail of renal transplantation is to combat progressive allograft injury through an elaborate understanding of the pathogenesis and evolution of effective therapies for the histopathologic entity called transplant glomerulopathy.

\section{Conclusions}

Technological breakthroughs in antibody characterization, coupled with the advent of emerging therapeutic modalities and antibody elimination protocols, have had a profound impact on the treatment options for highly sensitized patients. Each sensitized patient carries varying immunological risks. The need of the hour is to consider these risks and to formulate a rational and individualized treatment plan through effective communication and collaboration.

Acknowledgment Supported by Grants R01DK098431 and RC1 DK086731 from the National Institute of Diabetes and Digestive and Kidney Diseases (NIDDK) and by the Charles T. Bauer Foundation (R.M.)

\section{Compliance with Ethics Guidelines}

Conflict of Interest Hariharan S. Iyer declares that he has no conflict of interest.

Annette M. Jackson declares that she has no conflict of interest.

Robert A. Montgomery has received research grants from Alexion Pharmaceuticals, Inc. for studies involving the clinical use of eculizumab and from Viropharma, Inc. for Cinryze. He has also received grant support from Genzyme. He has been on the Astellas Scientific Advisory Board, the Gambro Advisory Board, and has been a consultant for iPierian Inc.

Human and Animal Rights and Informed Consent This article does not contain any studies with human or animal subjects performed by any of the authors. 


\section{References}

Papers of particular interest, published recently, have been highlighted as:

- Of importance

•• Of major importance

1. OPTN/SRTR annual report 2011. http://optn.transplant.hrsa.gov

2. Halloran PF et al. An integrated view of molecular changes, histopathology and outcomes in kidney transplants. Am J Transplant. 2010;10:2223-30.

3. Porcheray F et al. Expansion of polyreactive B cells cross-reactive to HLA and self in the blood of a patient with kidney graft rejection. Am J Transplant. 2012;12(8):2088-97.

4. Zachary AA, Montgomery RA, Leffell MS. Defining unacceptable HLA antigens. Curr Opin Organ Transplant. 2008;13:405-10.

5. Otten HG, Verhaar MC, Borst HP, van Eck M, et al. The significance of pretransplant donor-specific antibodies reactive with intact or denatured human leucocyte antigen in kidney transplantation. Clin Exp Immunol. 2013;173(3):536-43.

6. Idica AK, Kaneku HK, Sasaki N, Terasaki PI. Denatured and intact HLA specific antibody in kidney transplantation. Am J Transplant. 2009;9(S2):470.

7. Zachary AA, Sholander JT, Houp JA, Leffell MS. Using real data for a virtual crossmatch. Hum Immunol. 2009;70(8):574-9.

8. Zachary AA, Leffell MS. Detecting and monitoring human leukocyte antigen- specific antibodies. Hum Immunol. 2008;69(10):591604.

9. Christiaans MH, Roos R, Overhof-de Roos F, et al. Donor-specific antibodies after transplantation by flow cytometry: Relative change in fluorescence ratio most sensitive risk factor for graft survival. Transplantation. 1998;65:427-33.

10. Cecka JM. Calculated PRA, (CPRA): the new measure of sensitization for transplant candidates. Am J Transplant. 2009;10:26-9.

11. Eng SE, Leffell M. Histocompatibility testing after fifty years of transplantation. J Immunol Methods. 2011;369:1-21.

12. Leffell M. The calculated panel reactive antibody policy: an advancement improving organ allocation. Curr Opin Organ Transplant. 2011;16:404-9.

13. Chang D, Kobashigawa J. The use of calculated panel-reactive antibody and virtual cross match in heart transplantation. Curr Opin Organ Transplant. 2012;17:423-6.

14. Cecka JM, Reinsmoen NL, Leffell MS. Calculated PRA: Initial results show benefits for sensitized patients and a reduction in positive crossmatches. Am J Transplant. 2011;11:719-24.

15. Andrew SM, Titus JA, Coico R, et al. Purification of Immunoglobulin $\mathrm{M}$ and Immunoglobulin D. Curr Protoc Immunol. 2001; Chapter 2: Unit 2.9.

16. Zachary AA, Lucas DP, Detrick B, Leffell MS. Naturally occurring interference in Luminex assays for HLA-specific antibodies: characteristics and resolution. Hum Immunol. 2009;70(7):496-501.

17. Claas FH, Rahmel A, Doxiadis II. Enhanced kidney allocation to highly sensitized patients by the acceptable mismatch program. Transplantation. 2009;88:447-52.

18. Zachary AA, Montgomery RA, Leffell MS. Factors associated with and predictive of persistence of donor-specific antibody after treatment with plasmapheresis and intravenous immunoglobulin. Hum Immunol. 2005;66:364-70.

19. Montgomery RA, Warren D, Segev D, Zachary A. HLA incompatible renal transplantation. Curr Opin Organ Transplant. 2012;17: 386-92. An excellent review of the Johns Hopkins protocol for HLA-incompatible transplantation using desensitization, kidneypaired donation, or both.
20. Tambur AR, Leventhal JR, Friedewald JJ, Ramon DS. The complexity of human leukocyte antigen (HLA)-DQ antibodies and its effect on virtual crossmatching. Transplantation. 2010;90:1117-24.

21. Bagnasco SM, Zachary AA, Racusen LC, Montgomery RA, et al. Time Course of Pathologic Changes in Kidney Allografts of Positive Crossmatch HLA-Incompatible Transplant Recipients. Transplantation. 2013.

22. Haririan A, Nogueira J, Kukuruga D, et al. Positive cross-match living donor kidney transplantation: longer-term outcomes. Am J Transplant. 2009;9(3):536-42.

23. Bentall $\mathrm{A}$ et al. Five-year outcomes in living donor kidney transplants with a positive crossmatch. Am J Transplant. 2013;13(1):76-85.

24. Higgins R, Lowe D, Hathaway M. Human leukocyte antigen antibody incompatible renal transplantation: excellent medium-term outcomes with negative cytotoxic crossmatch. Transplantation. 2011;92:900-6.

25.• Montgomery RA, Lonze BE, King KE, et al. Desensitization in HLA- incompatible kidney recipients and survival. N Engl J Med. 2011;365:318-26. This matched-control analysis compares the outcomes of HLA-incompatible transplantation with other viable options for highly sensitized patients. Independent of the level of sensitization, HLA-incompatible transplantation offers significant survival benefit and higher transplantation rates.

26. Jordan SC, Tyan D, Stablein D, et al. Evaluation of intravenous immunoglobulin as an agent to lower allosensitization and improve transplantation in highly sensitized adult patients with end-stage renal disease: report of the NIHIG02 trial. J Am Soc Nephrol. 2004; 15:3256-62.

27. Vo AA, Lukovsky M, Toyoda M, et al. Rituximab and intravenous immunoglobulin for desensitization during renal transplantation. $\mathrm{N}$ Engl J Med. 2008;359:242-51.

28. Alachkar N, Lonze BE, Zachary AA, et al. Infusion of high-dose intravenous immunoglobulin fails to lower the strength of human leukocyte antigen antibodies in highly sensitized patients. Transplantation. 2012;94:165-71.

29. Lobashevsky AL, Higgins NG, Taber TE, et al. Analysis of antiHLA antibodies in sensitized kidney transplant candidates subjected to desensitization with intravenous immunoglobulin and rituximab. Transplantation. 2013;96(2):182-90.

30. Gentry SE, Montgomery RA, Segev DL. Kidney paired donation: fundamentals, limitations, and expansions. Am J Kidney Dis. 2011;57:144-51. This review summarizes the principles of kidneypaired donation and provides a thorough analysis of its outcomes, advantages and short- comings.

31. Segev DL, Gentry SE, Warren DS, et al. Kidney paired donation and optimizing the use of live donor organs. JAMA. 2005;293: 1883-90.

32. Garonzik-Wang JM, Sullivan B, Hiller JM, Montgomery RA. International kidney paired donation. Transplantation. 2013;96(7): e55-6.

33. Montgomery RA. Renal transplantation across HLA and ABO antibody barriers: integrating paired donation into desensitization protocols. Am J Transplant. 2010;10(3):449-57.

34. Montgomery RA, Zachary AA, Ratner LE, et al. Clinical results from transplanting incompatible live kidney donor/recipient pairs using kidney paired donation. JAMA. 2005;294:1655-63.

35.• Jackson A, Leffell M, Montgomery R, et al. A GPS for finding the route to \& transplantation for the sensitized patient. Curr Opin Organ Transplant. 2012;17:433-9. This paper provides an excellent review on the strategies for determining the most appropriate route to successful transplantation for highly sensitized patients. It also provides an in-depth analysis on the donor, patient and programspecific factors that influence decision-making.

36. Amico P, Hönger G, Mayr M, et al. Clinical relevance of pretransplant donor-specific HLA antibodies detected by singleantigen flow-beads. Transplantation. 2009;87:1681-8. 
37. Gloor JM, Winters JL, Stegall MD, et al. Baseline donor-specific antibody levels and outcomes in positive crossmatch kidney transplantation. Am J Transplant. 2010;10(3):582-9.

38. Hidalgo LG, Campbell PM, Halloran PF, et al. De novo donorspecific antibody at the time of kidney transplant biopsy associates with microvascular pathology and late graft failure. Am J Transplant. 2009;9(11):2532-41.

39. Wiebe C, Gibson IW, Nickerson PW, et al. Evolution and clinical pathologic correlations of de novo donor-specific HLA antibody post kidney transplant. Am J Transplant. 2012;12(5):1157-67.

40. Tait BD, Gebel HM, Nickerson PW, Zachary AA. Consensus guidelines on the testing and clinical management issues associated with HLA and non-HLA antibodies in transplantation. Transplantation. 2013;95(1):19-47. Concise guidelines and recommendations from the expert group regarding antibody monitoring for sensitized and non-sensitized patients.

41.• Montgomery RA, Leffell MS, Zachary AA. Transplantation of the sensitized patient: histocompatibility testing. Methods Mol Biol. 2013;1034:117-25. A comprehensive review of the obligations and responsibilities of the histocompatibility laboratory in antibody characterization before, during and after transplantation.

42. Woodle ES, Morrow W, Frazier E. Rapid reduction in donorspecific anti-human leukocyte antigen antibodies and reversal of antibody-medicated rejection with Bortezomib in pediatric heart transplant patients. Transplantation. 2012;93:319-24. This study provides evidence that Bortezomib, a proteasome inhibitor is an effective tool at depleting DSA and treating AMR.

43. Perry DK, Burns JM, Pollinger HS, et al. Proteasome inhibition causes apoptosis of normal human plasma cells preventing alloantibody production. Am J Transplant. 2009;9:201-9.

44. Stegall M, Diwan T, Raghavaiah S, Burns J. The impact of proteasome inhibition on alloantibody-producing plasma cells in vivo. Transplantation. 2011;91:536-41.

45. Ejaz NS, Woodle ES, Shields AR, et al. Randomized Controlled Pilot Study of B Cell-Targeted Induction Therapy in HLA Sensitized Kidney Transplant Recipients. Am J Transplant. 2013;13:3142-54

46. Colvin RB. Antibody-mediated renal allograft rejection: diagnosis and pathogenesis. J Am Soc Nephrol. 2007;18(4):1046-56.

47. McCaughan JA, O'Rourke DM, Courtney AE. Recurrent dense deposit disease after renal transplantation: an emerging role for complementary therapies. Am J Transplant. 2012;12(4):1046-51.

48. Lonze BE, Singer AL, Montgomery RA. Eculizumab and renal transplantation in a patient with CAPS. N Engl J Med. 2010;362(18):1744-5.

49. Locke JE, Magro CM, Singer AL, et al. The use of antibody to complement protein $\mathrm{C} 5$ for salvage treatment of severe antibodymediated rejection. Am J Transplant. 2009;9(1):231-5.

50.• Stegall MD, Diwan T, Raghavaiah S, et al. Terminal complement inhibition decreases antibody-mediated rejection in sensitized renal transplant recipients. Am J Transplant. 2011;11:2405-13. This study shows that complement inhibition therapy with Eculizumab significantly lowers the incidence of AMR in the early post-transplantation period in patients desensitized with plasmapheresis and IVIg.

51. Locke JE, Zachary AA, Haas M, et al. The utility of splenectomy as rescue treatment for severe acute antibody mediated rejection. Am J Transplant. 2007;7:842-6.

52. Kaplan B, Gangemi A, Thielke J, et al. Successful rescue of refractory, severe antibody mediated rejection with splenectomy. Transplantation. 2007;83:99-100.

53. Chen G, Sequeira F, Tyan DB. Novel C1q assay reveals a clinically relevant subset of human leukocyte antigen antibodies independent of immunoglobulin $\mathrm{G}$ strength on single antigen beads. Hum Immunol. 2011;72:849-58.

54. Sutherland SM, Chen G, Sequeira FA, Tyan DB, et al. Complementfixing donor- specific antibodies identified by a novel $\mathrm{C} 1 \mathrm{q}$ assay are associated with allograft loss. Pediatr Transplant 2011;16:12-7.

55.• Loupy A, Lefaucheur C, Vernerey D, et al. Complement-binding anti-HLA antibodies and kidney-allograft survival. N Engl J Med. 2013;369(13):1215-26.

56. Racusen LC, Leffell MS. C1q-binding antibodies in kidney transplantation. N Engl J Med. 2013;369(13):1266-7.

57. Opelz G. Non-HLA transplantation immunity revealed by lymphocytotoxic anti-bodies. Lancet. 2005;365(9470):1570-6.

58. Jackson AM, Lucas DP, Badders JL. A flow cytometric crossmatch test using endothelial precursor cells isolated from peripheral blood. Methods Mol Biol. 2013;1034:319-29.

59. Vermehren D, Sumitran-Holgersson S. Isolation of precursor endothelial cells from peripheral blood for donor-specific crossmatching before organ transplantation. Transplantation. 2002;74(11):1479-86.

$60 . \bullet$ Jackson AM, Kuperman MB, Montgomery RA. Multiple hyperacute $\&$ rejections in the absence of detectable complement activation in a patient with endothelial cell reactive antibody. Am J Transplant. 2012;12:1643-9. A report describing accelerated graft rejection in the presence of AECAs that were enriched for noncomplement-fixing subclasses IgG2 and IgG4. This highlights the need to recognize the varying clinical phenotypes associated with these antibodies.

61. Breimer ME, Rydberg L, Jackson AM, et al. Multicenter evaluation of a novel endothelial cell crossmatch test in kidney transplantation. Transplantation. 2009;87:549-56.

62. Jackson AM, Lucas DP, Melancon JK, et al. Clinical relevance and IgG subclass determination of non-HLA antibodies identified using endothelial cell precursors isolated from donor blood. Transplantation. 2011;92:54-60.

63. Zitzner JR, Shah S, Tambur AR, et al. A prospective study evaluating the role of donor-specific anti-endothelial crossmatch (XMONE assay) in predicting living donor kidney transplant outcome. Hum Immunol. 2013;74(11):1431-6.

64. Alheim M, AlMahri A, Nilsson J, et al. The outcome of the endothelial precursor cell crossmatch test in lymphocyte crossmatch positive and negative patients evaluated for living donor kidney transplantation. Hum Immunol. 2013;74(11):1437-44.

65. Hiemann N, Meyer R, Wellnhofer E, et al. Non-HLA antibodies targeting vascular receptors enhance alloimmune response and microvasculopathy after heart transplantation. Transplantation. 2012;94:919-24.

66. Dragun D, Müller DN, Bräsen JH, et al. Angiotensin II type 1receptor activating antibodies in renal-allograft rejection. $\mathrm{N}$ Engl J Med. 2005;352:558-69.

67. Giral M, Foucher Y, Dufay A, Soulillou JP, et al. Pretransplant sensitization against angiotensin II type 1 receptor is a risk factor for acute rejection and graft loss. Am J Transplant. 2013;13(10):2567-76.

68. Alachkar N, Gupta G, Montgomery RA. Angiotensin antibodies and focal segmental glomerulosclerosis. N Engl J Med. 2013;368: 971-3. This report describes an interesting case of AMR and new onset collapsing FSGS due to antibodies against AT1R. Treatment with plasmapheresis, IVIg and losartan resulted in resolution of the histological lesions.

69. Lucas DP, Leffell MS, Zachary AA. Tetramer staining for the detection of HLA-specific B cells. Methods Mol Biol. 2013;1034:313-8.

70. Zachary AA, Kopchaliiska D, Montgomery RA, et al. HLAspecific B cells: I. A method for their detection, quantification, and isolation using HLA tetramers. Transplantation. 2007;83(7): 982-8.

71. Zachary AA, Kopchaliiska D, Montgomery RA, et al. HLA-specific B cells: II. Application to transplantation. Transplantation. 2007;83(7): 989-94.

72. Zachary A, Lucas D, Montgomery RA, et al. Rituximab prevents an anamnestic response in patients with cryptic sensitization. Transplantation. 2013;95:701-4. 